A Propspective pilot study to review the impact of "SnooZeal" on CPAP utilistation data in patients with moderate to severe OSA

C Saxby, H Zhang, A Eaton, D Wright, B Kotecha

Royal National Throat Nose and Ear Hospital, London, UK

\title{
Introduction and Aims
}

SnooZeal is an anti-snoring device, which aims to stimulate the tongue muscles during wakefulness using an electrical current via electrodes that are placed above and below the tongue. This is thought to enhances the tongue muscle tone in general and therefore reduces the impact of loss of muscle tone during sleep that causes airway obstruction and snoring.

Our aim was to see if the use of SnooZeal improves CPAP utilisation data in patients with moderate to severe OSA and therefore CPAP compliane and efficacy.

Mouth piece: designed to fit around the tongue like a glove

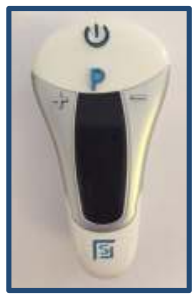

Remote control unit used to manage the control unit's functions

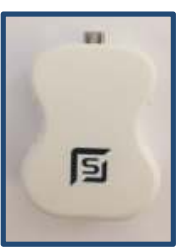

Control unit: a rechargeable unit that attaches to the mouth piece via a mini-USB connection

\section{Methods}

Five patients were recruited and instructed to use SnooZeal twice daily for a 6 week period. CPAP utilisation data was assessed for: average hours of use per night, average pressure required, $\mathrm{AHI}$ and the patients completed an Epworth Sleepiness Scale. The data was reviewed during a pre-treatment period ( 6 weeks) and treatment period (6 weeks).

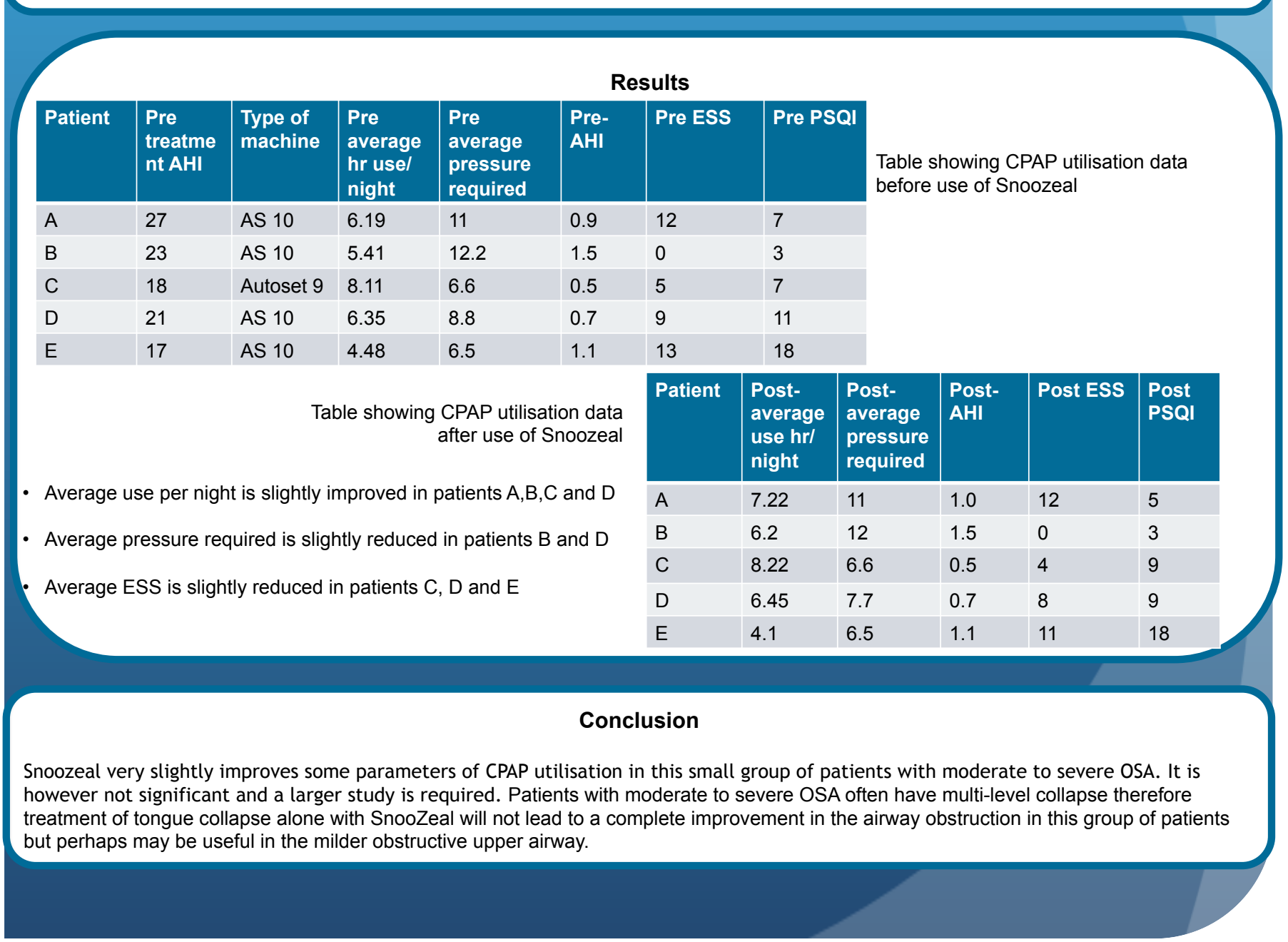

